\title{
Identificación del modelo de un motor de DC mediante métodos gráficos y métodos paramétricos.
}

Diego Verdugo O., Paúl Mata Q., Alvarito Coronel G., Javier González R. 


\title{
Identificación del modelo de un motor de DC mediante métodos gráficos y métodos paramétricos \\ Identification of the model of a DC engine through graphic methods and parametric methods
}

\author{
Diego Verdugo O. ${ }^{(1)}$ Paúl Mata Q. ${ }^{(2)}$ Alvarito Coronel G. ${ }^{(3)}$ Javier González R. ${ }^{(4)}$ \\ Unidad Académica de Ingeniería, Industria y Construcción ${ }^{(1)}$ \\ Universidad Católica de Cuenca (UCACUE) \\ Campus Luis Cordero "El Grande", Av. Ernesto "Che” Guevara y Av. 16 de Abril \\ Azogues - Ecuador \\ deverdugoo@ucacue.edu.ec ${ }^{(1)}$
}

\begin{abstract}
Resumen
Este artículo presenta el estudio para conseguir el modelo de un motor-generador (planta), se emplean métodos gráficos o paramétricos que nos servirán como herramientas para representar dicho sistema de forma matemática. Se han adquirido muestras a través de una DAQ del comportamiento del sistema ante un escalón y una señal PRBS, los datos obtenidos se procesaron a través del software MATLAB y el toolbox IDENT, donde por medio de criterios de selección se obtiene un modelo ARX. Las técnicas estudiadas fueron: identificación paramétrica con la aplicación de una señal PRBS y estructura de modelo ARX e identificación no paramétrica con la aplicación de métodos gráficos. Luego se realiza una comparación entre los datos reales con los diferentes métodos aplicados y se obtiene que las respuestas son muy similares. La identificación de sistemas nos sirve para construir modelos matemáticos de sistemas dinámicos basándonos en los datos observados, con lo cual se tiene un conjunto de técnicas para levantar un proceso.
\end{abstract}

Palabras claves: Identificación de sistemas, método gráfico, método de primer orden puro, método ARX.

\begin{abstract}
This article presents the study to obtain the model of an engine-generator system (plant), we use graphical or parametric methods that will serve as tools to represent said system in a mathematical way. The signs were acquired through a DAQ system behavior before a step and PRBS signal, the data was processed through MATLAB software and IDENT toolbox, where through selection criteria described subsequently obtained an ARX model. The techniques studied were: parametric identification with the application of a PRBS signal and ARX model structure and nonparametric identification with the application of graphic methods. Then a comparison is made between the actual data with the different methods applied and it is obtained that the answers are very similar. The identification of systems serves to construct mathematical models of dynamic systems based on observed data, which has a set of techniques to build a process.
\end{abstract}

Keywords: Identification of systems, graphic method, pure first order method, ARX method. 


\section{Introducción}

La identificación de sistemas se define ampliamente como la deducción de los modelos matemáticos del sistema, a partir del análisis de datos experimentales, mediciones y observaciones. Su base teórica se sostiene en parte por la teoría de sistemas dinámicos y señales de naturaleza estocástica, también por métodos y algoritmos matemáticos para la correcta estimación de los parámetros involucrados [1].

El proceso de pasar de los datos observados a un modelo matemático es fundamental en la ciencia y la ingeniería, donde se requiere de modelos cada vez más exactos para fines de análisis, predicción, simulación, diseño y control. En el área de control, este proceso se denomina identificación del sistema y el objetivo es encontrar modelos matemáticos dinámicos (ecuaciones diferenciales) a partir de señales de entrada y salida observadas. Sus características básicas son, sin embargo, comunes con los procesos generales de construcción de modelos en la estadística y otras ciencias [2].

Muchas de las ocasiones, los modelos no pueden ser obtenidos tan fácilmente a partir de las leyes que rigen cada proceso. Por lo tanto, la construcción de modelos para sistemas desconocidos juega un papel determinante para ofrecer los métodos necesarios para obtener de manera relativamente sencilla los modelos matemáticos buscados con un alto grado de exactitud [1]. Existen modelos lineales conocidos como ARX y ARMAX, y entre los modelos no lineales tenemos, por ejemplo, redes neuronales artificiales (ANN). Estos modelos necesitan simular el comportamiento real en los casos en que existe un conocimiento previo limitado de la estructura del sistema [3].

\section{Identificación de sistemas}

Para realizar el análisis de la planta, el sistema o el proceso, se requiere un modelo matemático que lo represente. De manera general, para el proceso de identificación, se tiene los siguientes pasos: [4]

1. Obtención de datos de entrada-salida: mediante la aplicación de una señal de entrada registramos la evolución de sus entradas y salidas durante un intervalo de tiempo.

2. Tratamiento previo de los datos registrados: preparar los datos para facilitar y mejorar el proceso de identificación.

3. Elección de la estructura del modelo: se facilita en gran medida si se tiene un cierto conocimiento sobre las leyes físicas que rigen el proceso.

4. Obtención de los parámetros del modelo: se procede a la estimación de los parámetros de la estructura que mejor se ajustan a la respuesta del modelo con los datos de entrada-salida obtenidos experimentalmente.
5. Validación del modelo: es un proceso vital, si el modelo obtenido satisface el grado de exactitud requerido para la aplicación.

Si el modelo no es válido:

a) El conjunto de datos entrada-salida no proporciona suficiente información sobre la dinámica del sistema.

b) La estructura escogida no es capaz de proporcionar una buena descripción del modelo.

c) El criterio de ajuste de parámetros seleccionado no es el más adecuado.

\section{Descripción de la planta}

El funcionamiento de la planta se basa en proporcionarle una alimentación (referencia) al motor DC, de acuerdo con este valor de voltaje suministrado este empieza a girar a una cierta velocidad, lo cual hace que actúe el generador debido a que se encuentran acoplados mediante una banda y el generador que tiene similares características a la del motor nos da una señal de salida de voltaje. El motor DC genera 7V a 1000 rpm.

Al conocer el principio de funcionamiento de la planta de nuestro proyecto (Figura 1), se van a aplicar dos métodos de identificación de sistemas, el primero que es un método gráfico y el segundo que es un método paramétrico, los cuales serán explicados posteriormente y de esta manera verificar que estos modelos se comportan igual que la planta implementada.

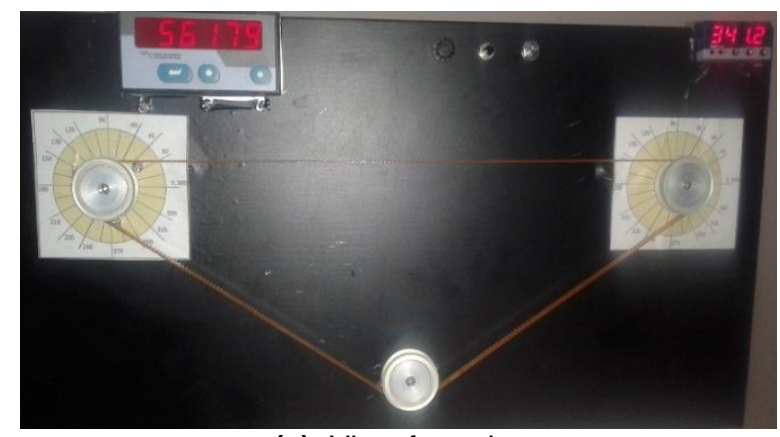

(a). Vista frontal

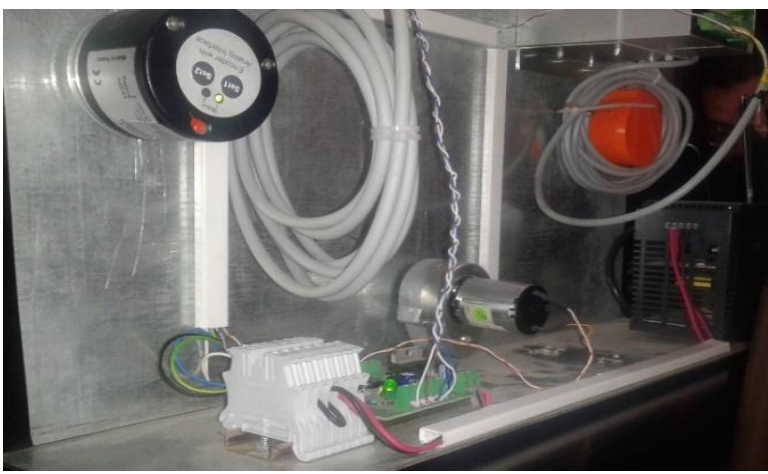

(b). Vista posterior

Figura 1. Motor DC con generador (Planta) 


\section{Métodos de identificación}

\subsection{Método gráfico: Identificación basada en la respuesta al escalón a lazo abierto}

Este método se caracteriza por determinar los parámetros del modelo de una forma gráfica. Consiste en aplicar una entrada en forma de escalón sobre un sistema en equilibrio y observar la respuesta, mediante el análisis de la salida se obtiene la función de transferencia, que pretende ser lo más cercano al comportamiento del proceso [5].

La señal de prueba más utilizada es el escalón, en la práctica sólo se puede conseguir de forma aproximada ya que es imposible lograr un cambio brusco de una variable en un tiempo infinitesimal, sin embargo, se puede considerar como una señal válida si la constante de tiempo de la señal real es menor que la décima parte de la menor constante de tiempo que se quiere determinar en la identificación [6].

4.1.1. Método Gráfico: Método de primer orden puro. Se expresa en la ecuación (1), la función de transferencia para este tipo de sistemas:

$$
\mathrm{G}(\mathrm{s})=\frac{\mathrm{K}}{\tau \mathrm{s}+1}
$$

donde:

$\mathrm{K}=$ ganancia del sistema $=\frac{\text { señal entrada }}{\text { señal salida }}=\frac{\Delta \mathrm{y}}{\Delta \mathrm{u}}$

$\tau=$ constante de tiempo

Se necesitan estimar la ganancia (K), y la constante de tiempo $(\tau)$.

La Figura 2 ilustra la respuesta de este método, la misma que no presenta sobreoscilaciones, lo que significa que nunca llegan al valor exacto de la consigna, es decir; son sistemas relativamente lentos [5].

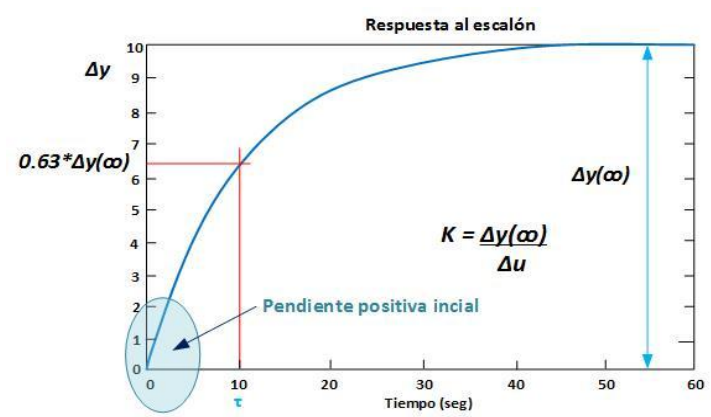

Figura 2. Sistema de primer orden puro [5]

El valor de la constante de tiempo se obtiene de la gráfica, para lo cual se observa el tiempo correspondiente a un valor del $0.63 \% \Delta y$.
4.1.2. Método Gráfico: Método de la tangente. La función de transferencia de este tipo de sistemas se formuló en la ecuación (1) y la ganancia del sistema (K) se expresó en la ecuación (2).

La Figura 3 muestra la respuesta de este método, el mismo que no presenta sobreoscilaciones, lo cual significa que nunca llega al valor exacto de la consigna [5].
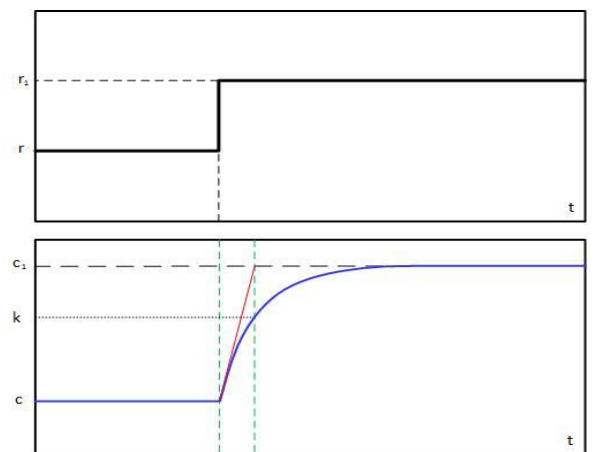

Figura 3. Escalón de entrada y respuesta del sistema de primer orden [6]

El valor de la constante $\tau$ se obtiene al proyectar perpendicularmente al eje del tiempo el punto donde corta la recta tangente a la respuesta con la recta $\mathrm{y}=$ $\Delta \mathrm{y}($ ? $)$.

\subsection{Método paramétrico: Identificación por mínimos cuadrados}

Utilizando secuencias ordenadas, una de entrada $\left(u_{k}\right)$ y una de salida $\left(y_{k}\right)$, se ajusta un modelo ARX a la operación del sistema dinámico a través de la minimización cuadrática del error $\left(e_{k}=y_{k}-\hat{y}_{k}\right)$. La Figura 4 muestra un esquema general de la aplicación de este modelo [7].

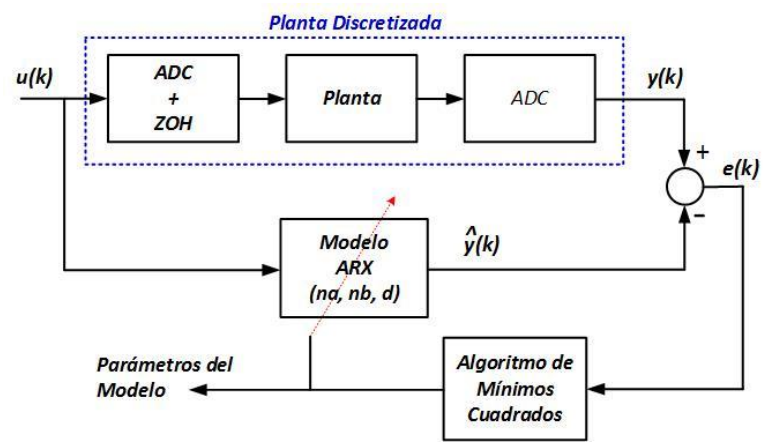

Figura 4. Diagrama de bloques del sistema [7]

4.2.1. Método paramétrico: Método de mínimos cuadrados - ARX. Este método trata de ajustar un modelo ideal de estructura auto-regresiva con variable exógena a los datos obtenidos en el funcionamiento real del sistema. [6] 
$y_{k}-a_{2} y_{k-2}-\ldots-a_{n a} y_{k-n a}=b_{1} u_{k-1}+\ldots+b_{n b} u_{k-n b}+\eta_{k}$ $y_{k}\left(1-a_{1} z^{-1}-\ldots-a_{n a} z^{-n a}\right)=\left(b_{1} z^{-1}+\ldots+b_{n b} z^{-n b}\right) u_{k}+\eta_{k}$

Esto da lugar a un sistema de ecuaciones donde las incógnitas $a$ y $b$ serán los coeficientes de la función de transferencia.

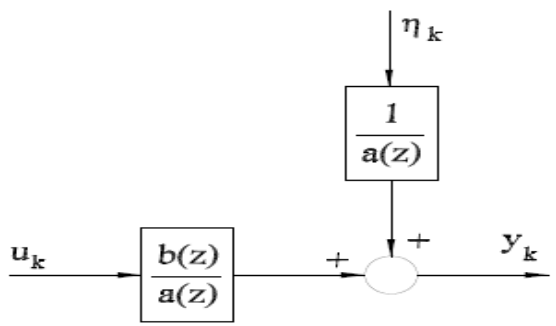

Figura 5. Diagrama de la salida del sistema de forma general. [7]

$$
y_{k}=\frac{b(z)}{a(z)} u_{k}+\frac{1}{a(z)} \eta_{k}
$$

El error viene dado por la ecuación (4):

$$
e_{k}=y_{k}-a_{1} y_{k-1}-\ldots-a_{n a} y_{k-n a}-b_{1} u_{k-1-d}-\ldots-b_{n b} u_{k-n b-d}
$$

Las ecuaciones se empiezan a generar a partir de:

$$
k=n m, \ldots, N \text { у } n m=\max [n a, n b+d]
$$

donde:

- na: Representa el número de pasos del tiempo de la salida en el pasado, orden del polinomio a(z).

- $\quad n b$ : Es el número de pasos del tiempo de la entrada en el pasado, orden del polinomio $\mathrm{b}(\mathrm{z})$.

Por lo tanto las ecuaciones (5), (6) y (7) enuncian las expresiones que se implementan en este método:

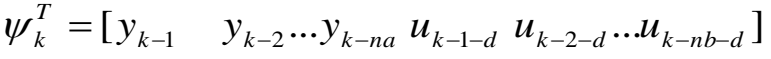

$$
\begin{aligned}
& \theta_{N}=\left[\begin{array}{llll}
a_{1} & a_{2} \ldots a_{b} & b_{1} & b_{2} \ldots b_{n b}
\end{array}\right] \\
& y_{k}=\psi_{k}^{T} \theta_{N}+e_{k}
\end{aligned}
$$

\section{Proceso de identificación de la planta}

Para el proceso de identificación se realiza la adquisición de datos usando el software LabView, con el cual se obtendrán los datos de entrada y salida de la planta que se está modelando mediante la tarjeta de National Instruments DAQ NI USB 6112, luego estos datos obtenidos se procesan en el software MATLAB, ya sea en la generación de las señales y en la aplicación de los métodos. A continuación, se coloca el proceso para la identificación de la planta.

\subsection{Obtención de datos entrada-salida}

5.1.1. Métodos gráficos: Primer orden puro y tangente (Respuesta al escalón en lazo abierto). En este método se usa una señal escalón a la entrada de la planta, esta señal debe ser lo suficientemente grande para que la solución del proceso cambie significativamente respecto del ruido de medida del sensor utilizado. La Figura 6 muestra que en nuestro caso la amplitud de la señal de excitación es de $8 \mathrm{~V}$, dicha señal fue generada en LabView mediante el archivo ESCALON.vi.

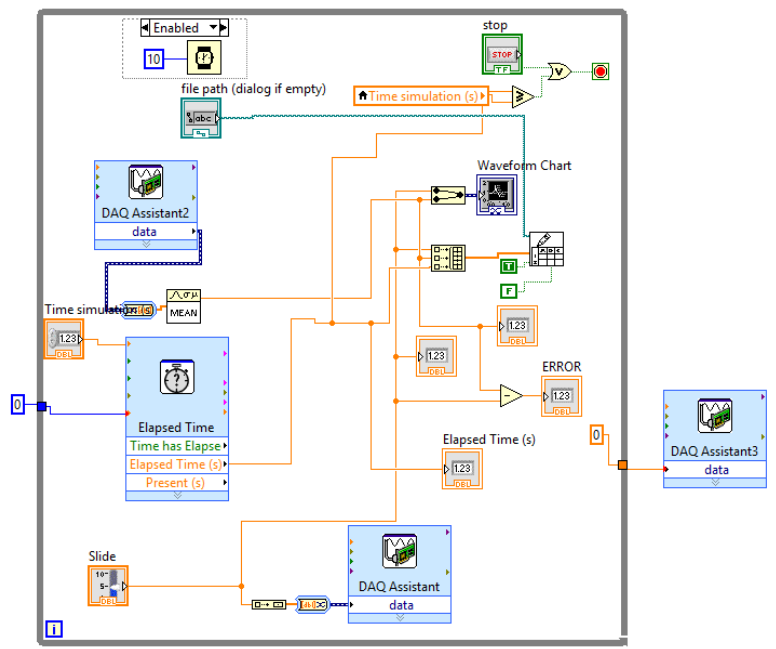

(a). Diagrama de bloques

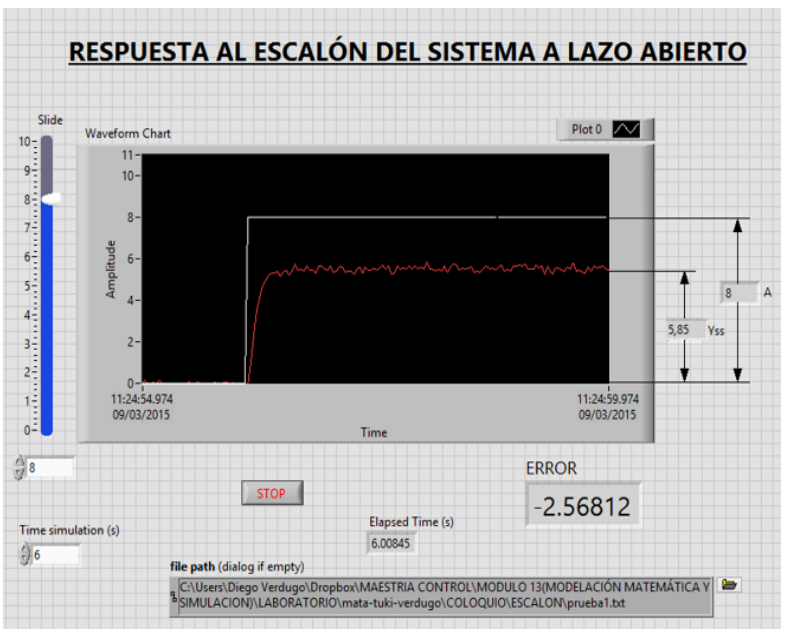

(b). Panel frontal

Figura 6. Archivo ESCALON.vi

Dichos datos son guardados en un archivo prueba1.txt. En los cuales constan la entrada (uk), la salida (yk) y el tiempo (t).

La Figura 7 muestra la gráfica en MATLAB de los datos yk (salida). 


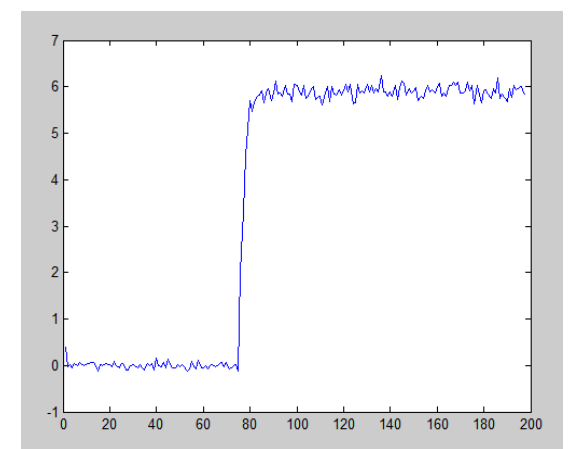

Figura 7. Grafica de la salida $y k$

5.1.2. Método Paramétrico: Identificación por mínimos cuadrados. Este método utiliza en cambio para la entrada una señal PRBS. Las secuencias binarias pseudoaleatorias (PRBS) son secuencias de pulsos rectangulares, modulados en ancho de pulso, que se aproximan a una señal discreta de ruido blanco, la cual se utiliza para el método de mínimos cuadrados-ARX. Dicha señal tiene una amplitud entre 2.4 y 4.1 con una duración de $7 \mathrm{~s}$. Se escogió dicho tiempo debido que al realizar varias pruebas se observó que es suficiente para obtener una identificación óptima del modelo que corresponda al de la planta. La Fig. 8 nos muestra la señal generada en LabView mediante el archivo IDENTIFICACION_PRBS.vi.

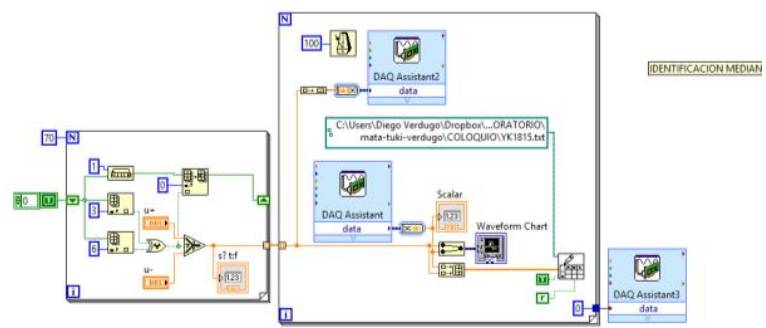

(a). Diagrama de bloques

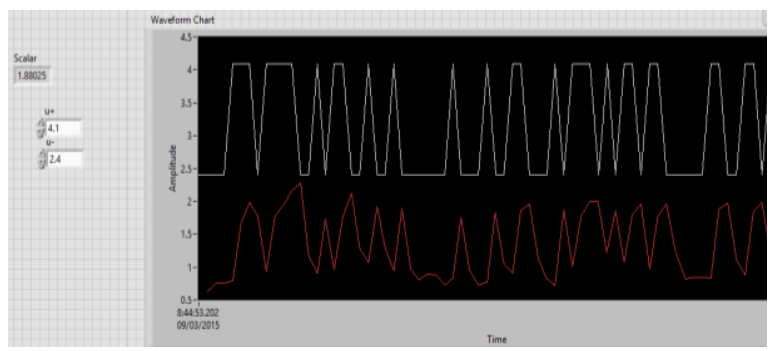

(b). Panel frontal

Figura 8. Archivo IDENTIFICACION_PRBS

Dichos datos son guardados en un archivo yk.txt. En los cuales constan la entrada (uk) y la salida (yk).

La Figura 9 muestra la gráfica en MATLAB de los datos yk de salida.

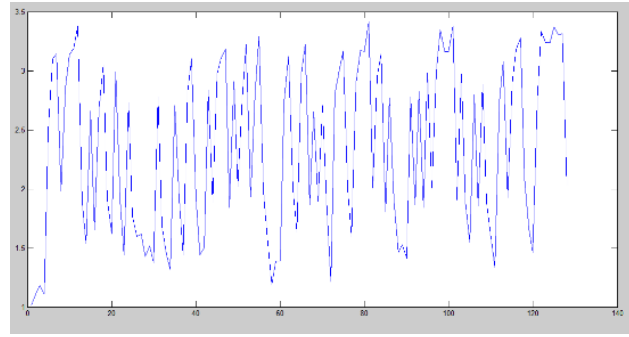

Figura 9. Grafica de la salida yk

5.2. Tratamiento previo de los datos de entrada y salida

5.2.1. Métodos Gráficos: Primer Orden Puro y Tangente (Respuesta al escalón en lazo abierto). Cargamos los datos obtenidos en MATLAB mediante el archivo CARGAR_DATOS_ESCALON.m el mismo que colocamos a continuación:

clc; clear all;

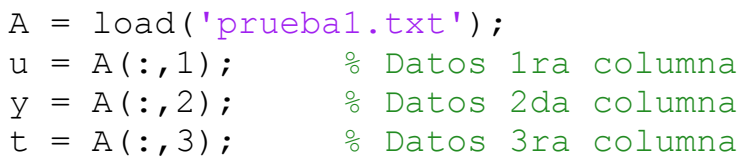

Una vez registrados los datos hay que corregirlos antes de iniciar la identificación del modelo, por tanto, se trata de preparar los datos para facilitar y mejorar el proceso de identificación. Como se pudo observar en la Figura 7 existen datos que no son relevantes por lo que se determina los datos que son necesarios para nuestra identificación. La Figura 10 indica que los datos a ser utilizados son desde la muestra 75 en adelante.

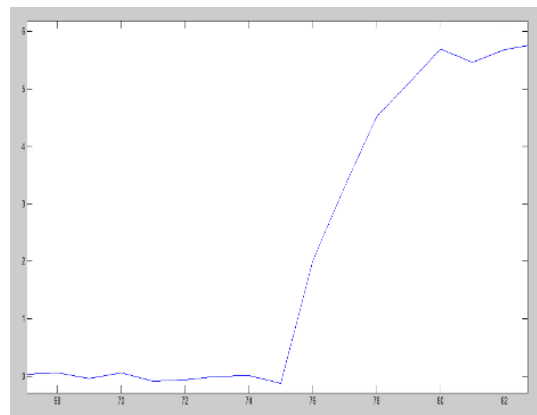

(a). Identificación datos válidos.

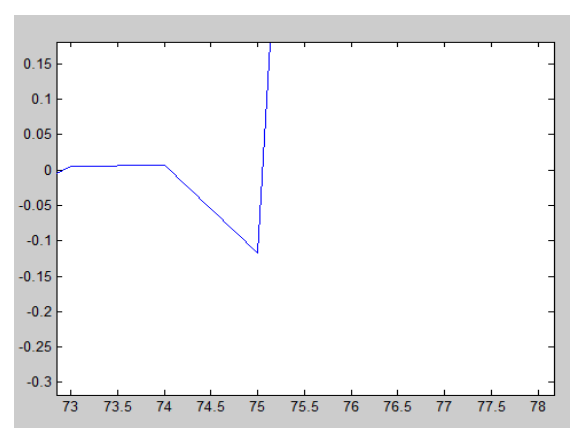

(b). Identificación datos válidos muestra 75

Figura 10. Identificación datos válidos 
Una vez determinada desde qué muestra se va a utilizar, se realiza una depuración de los datos para obtener solamente los necesarios, esto se realiza con las siguientes instrucciones de MATLAB:

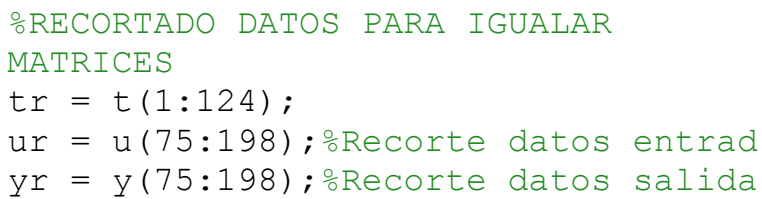

La Figura 11 presenta los datos de la salida $y r$ recortados en MATLAB.

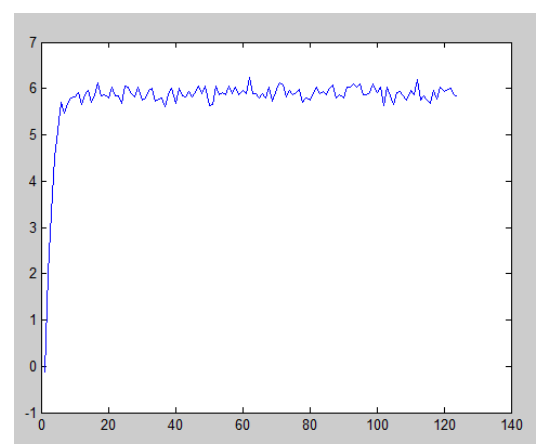

Figura 11. Grafica de la salida recortada (yr)

5.2.2. Método Paramétrico: Identificación por mínimos cuadrados. Cargamos los datos en MATLAB mediante el archivo CARGAR_DATOS.m el mismo que consta de las siguientes instrucciones:

Clc;

clear all;

$A=\operatorname{load}(' y k \cdot t x t$ ') ;

$\mathrm{yk}=\mathrm{A}(:, 1) ; \quad$ o Datos 1ra columna

$\mathrm{uk}=\mathrm{A}(:, 2) ; \quad$ o Datos 2 da columna

Para este método los datos obtenidos para la salida $y k$ son todos válidos debido a que la señal de entrada PRBS en su contenido frecuencial, se aproxima a un ruido blanco discreto.

\subsection{Elección de la estructura y obtención de los parámetros del modelo}

Para este paso se va a estimar en MATLAB los métodos que tengan una estructura que represente el comportamiento de la planta real.

5.3.1. Método Gráfico Primer Orden Puro. Por inspección de la salida $y r$ en la Figura 11., se puede determinar que la respuesta no presenta sobreoscilación y tampoco tiene retardo por lo que se puede asumir que el método a usar es el de primer orden puro cuya función de transferencia se expresó en la ecuación (1), también se puede determinar el valor de $\Delta \mathrm{y}(\infty)=5.89$, la Figura 12 demuestra lo expuesto anteriormente.

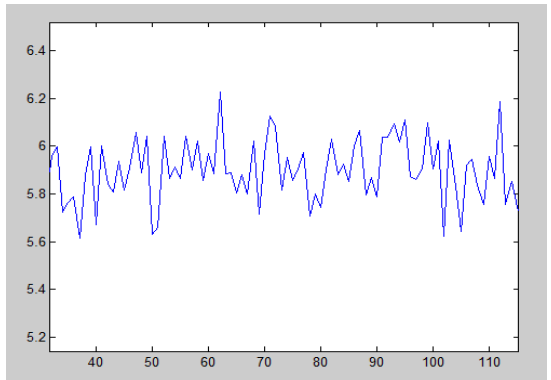

Figura 12. Gráfica de $\Delta y(\square)$

Usando la ecuación (2) obtenemos el valor de K:

$$
\mathrm{K}=\frac{\Delta \mathrm{y}(\infty)}{\Delta \mathrm{u}}=\frac{5.89}{8}=\mathbf{0 . 7 3 6 2 5}
$$

Ahora se calcula el valor de la constante de tiempo $(\tau)$ la misma que se obtiene sobre la gráfica, para ello se observa el tiempo correspondiente a un valor de $0.63 \Delta \mathrm{y}(\infty)$, en este caso se tendría que el valor de $0.63 \Delta \mathrm{y}(\infty)=0.63(5.89)=3.7107 \mathrm{~V}$, luego en la gráfica se determina el valor de la constante de tiempo $\tau=$ $0.0643 \mathrm{~s}$, este valor se indica en la Figura 13.

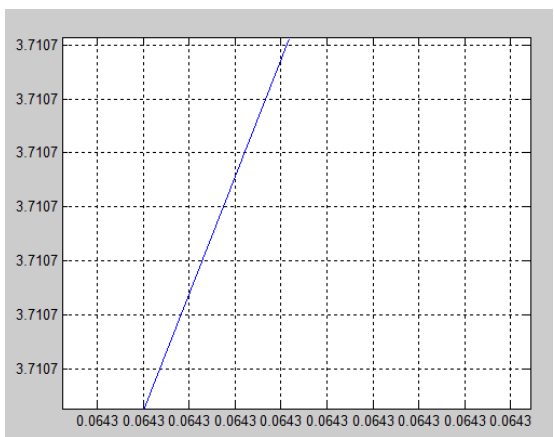

Figura 13. Grafica de $\tau$ cuando $0.63 \Delta \Delta y(\square)$

$$
0.63 \Delta y(\infty)=3.7107 \rightarrow \tau=\mathbf{0 . 0 6 4 3}
$$

Con los parámetros encontrados la función de transferencia representada por la ecuación (1) quedaría de la siguiente manera:

$$
\mathrm{Gp}(\mathrm{s})=\frac{0.73625}{0.0643 \mathrm{~s}+1}
$$

La Figura 14 presenta los resultados de la simulación de la función de transferencia realizada en simulink de MATLAB, con lo que se obtiene la señal de respuesta a un escalón de $8 \mathrm{~V}$ de amplitud.

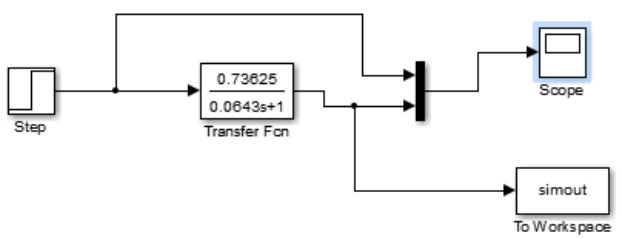

(a). Esquema de simulación 


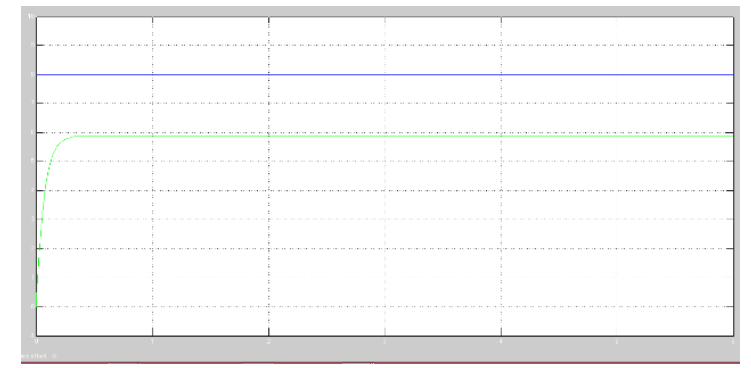

(b). Resultado de la identificación

Figura 14. Simulación en MATLAB.

La Figura 15 nos presenta la comparación entre el resultado alcanzado mediante este método con los datos de salida obtenidos al generar un entrada escalón de $8 \mathrm{~V}$ $(y r)$.

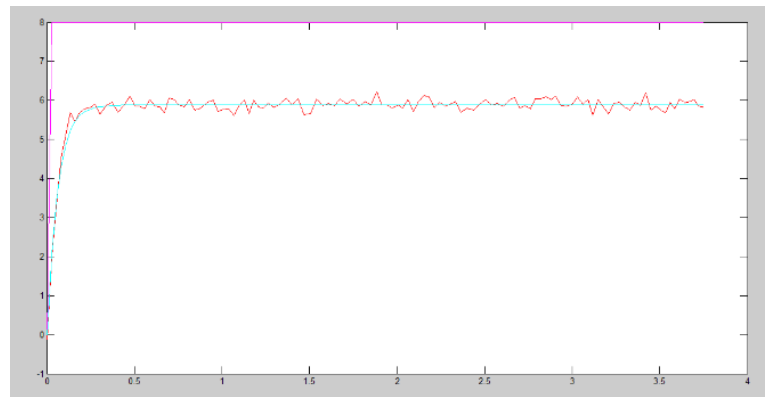

Figura 15. Comparación de resultados

Como se puede observar la salida yr y la señal de identificación de la planta son muy similares.

5.3.2. Método gráfico: Método de la tangente. Observando la salida $y r$ en la Figura 11, se puede establecer que la respuesta no presenta sobreoscilación ni retardo por lo que podemos asumir que el método a usar es el de la tangente cuya función de transferencia se expresó en la ecuación (1), también podemos determinar el valor de $\Delta y(\infty)=5.8$, la Figura 16 indica lo expuesto anteriormente.

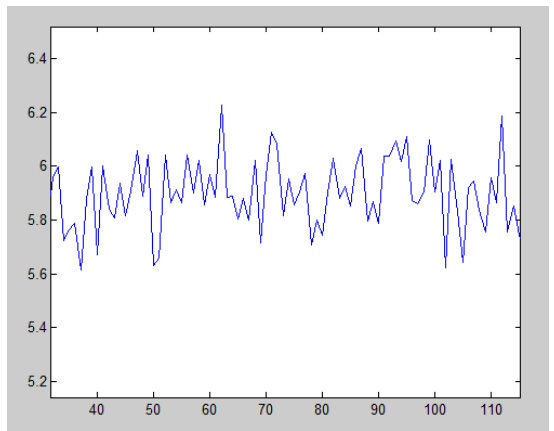

Figura 16. Grafica de $\Delta y(\square)$

Usando la ecuación (2) obtenemos el valor de K:

$$
\mathrm{K}=\frac{\Delta \mathrm{y}(\infty)}{\Delta \mathrm{u}}=\frac{5.8}{8}=\mathbf{0 . 7 2 5}
$$

A continuación se calcula el valor de la constante de tiempo $(\tau)$ la misma que se obtiene sobre la gráfica, para ello se observa el tiempo correspondiente al corte entre la recta tangente a la salida y la recta $\mathrm{y}=\Delta \mathrm{y}(\infty)$, luego en la gráfica se determina el valor de la constante de tiempo $\tau=0.079 \mathrm{~s}$, este valor se indica en la Figura 17 .

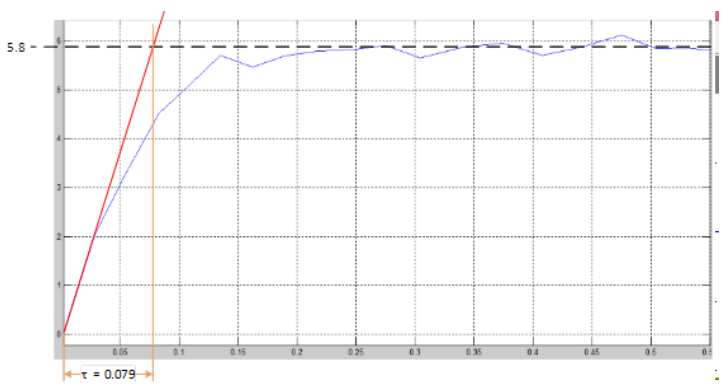

Figura 17. Gráfica de la constante de tiempo $\tau$

Con los parámetros calculados la función de transferencia representada por la ecuación (1) quedaría de la siguiente manera:

$$
\mathrm{Gp}(\mathrm{s})=\frac{0.725}{0.079 \mathrm{~s}+1}
$$

La Figura 18 presenta los resultados de la simulación de la función de transferencia realizada en simulink de MATLAB, con lo que se obtiene la señal de respuesta a un escalón de $8 \mathrm{~V}$ de amplitud.

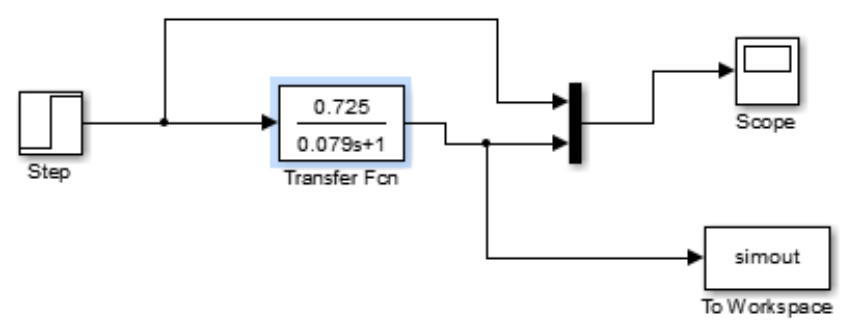

(a). Esquema de Simulación

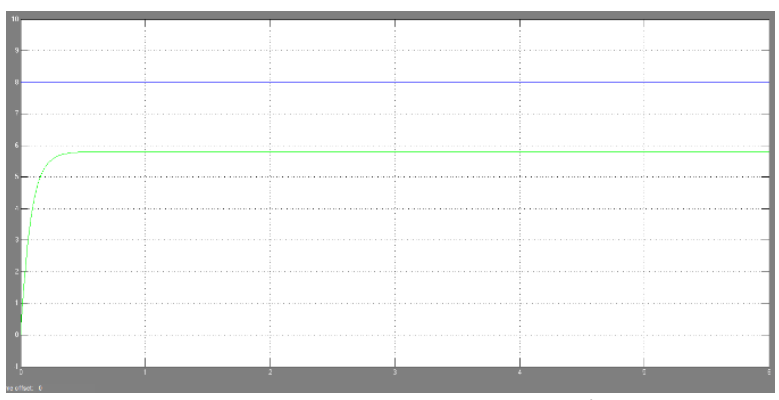

(b). Resultado de la identificación

Figura 18. Simulación en MATLAB.

La Figura 19 expone la comparación entre el resultado alcanzado mediante este método gráfico con los datos obtenidos de la salida al generar una entrada escalón de $8 \mathrm{~V}$ (yr). 


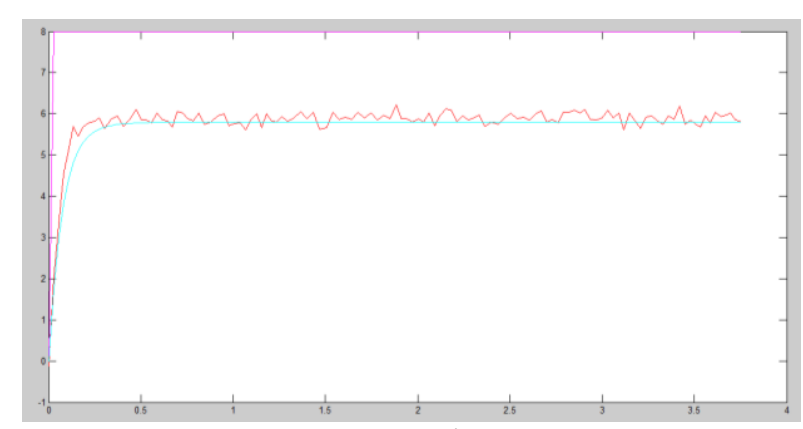

Figura 19. Comparación de resultados

5.3.3. Método paramétrico: Identificación por mínimos cuadrados. Con los datos cargados tanto de entrada (uk) como de salida (yk), se procede a utilizar la herramienta IDENT de MATLAB.

La Figura 20 muestra los datos de entrada y salida cargados en la herramienta ident.

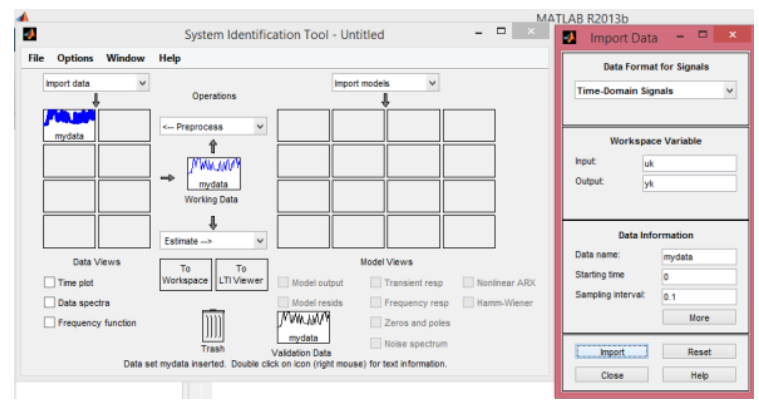

Figura 20. Herramienta IDENT de MATLAB

Luego se procede a realizar la estimación usando modelos polinomiales (Polynomial Models) con la estructura ARX de diferentes órdenes como por ejemplo [lll 110$]$; [ $\left.\begin{array}{lll}1 & 2 & 0\end{array}\right]$; [ $\left.\begin{array}{lll}2 & 1 & 0\end{array}\right]$, etc. La Figura 21 presenta lo expuesto anteriormente.

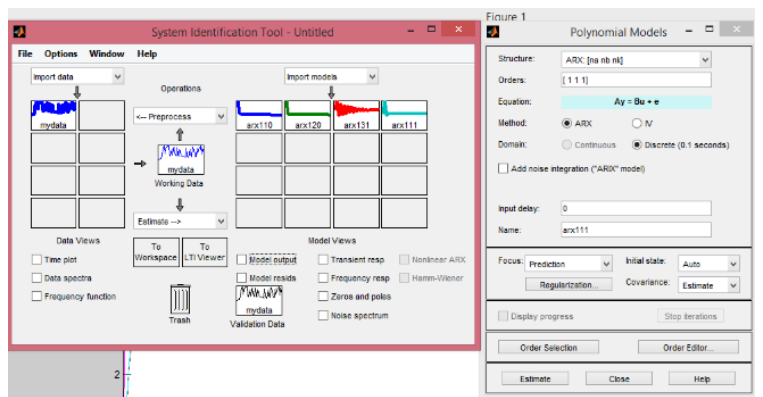

Figura. 21. Estimación de diferentes modelos usando modelos polinomiales (ARX).

Luego se determina qué modelo se asemeja más a nuestra planta, después de un análisis de las diferentes gráficas mostradas en la Figura 22 que permite realizar esta herramienta.

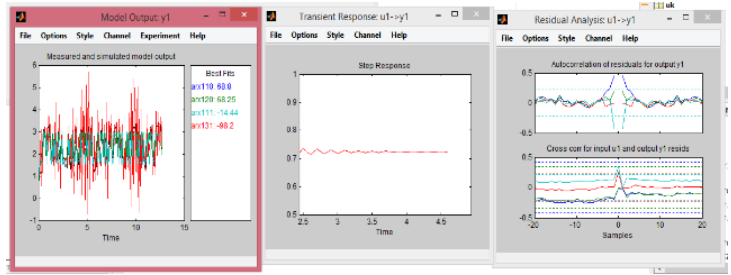

Figura 22 Respuestas de los diferentes modelos

Se escoge ARX110 debido a que tiene un porcentaje de $68.8 \%$ de semejanza con la salida real, siendo este el modelo con más alto porcentaje que el resto de modelos con los que se realizó la estimación; también se realizó un análisis de los residuos y este modelo cumple con los rangos establecidas para este estudio.

A continuación, se envía el modelo ARX110 al workspace de MATLAB arrastrando el modelo seleccionado hacia To Workspace en la herramienta IDENT.

Después se carga el archivo de MATLAB IDENTIFICACION.m para encontrar la función de transferencia de nuestro modelo ARX110, el código de dicho archivo es el siguiente:

○ IDENTIFICACIÓN DE LA F.T.

clc;

num $=\operatorname{arx} 110 . B$

den $=\operatorname{arx} 110 . \mathrm{A}$

\% Dominio de $z$ a $s$

$\mathrm{Ts}=0.1$;

$\mathrm{H}=\mathrm{t} f(\mathrm{num}, \mathrm{den}, \mathrm{Ts})$

$\mathrm{HC}=\mathrm{d} 2 \mathrm{C}(\mathrm{H})$

Obteniendo la siguiente función de transferencia de primer orden:

$$
\mathrm{Gp}(\mathrm{s})=\frac{13.99}{\mathrm{~s}+19.03}
$$

La Figura 23 presenta la respuesta de la simulación de la función de transferencia realizada en simulink.

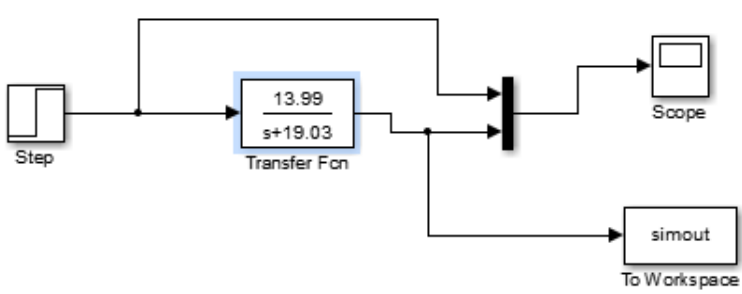

(a). Esquema de simulación 


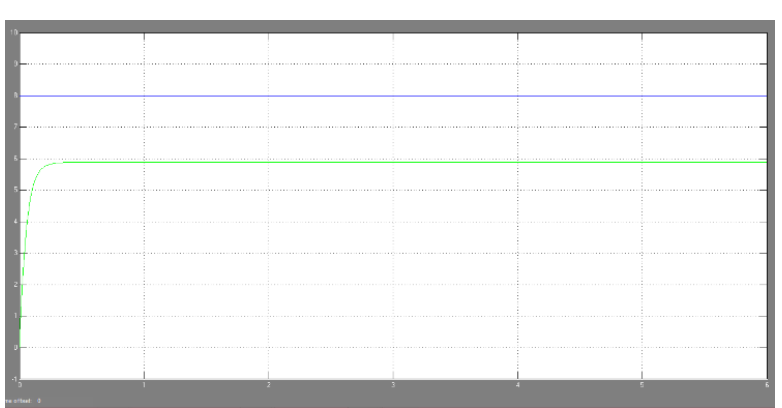

(b). Resultado de la identificación.

Figura 23. Simulación en MATLAB.

La Figura 24 nos muestra la comparación entre el resultado alcanzado mediante este método paramétrico con los datos de salida obtenidos al generar una entrada escalón de 8V (yr).

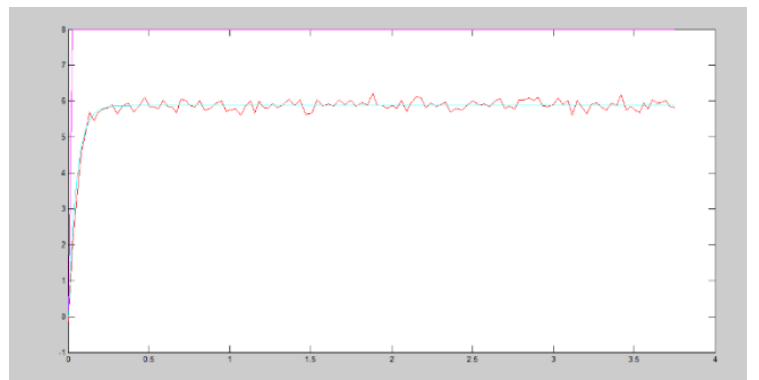

Figura 24. Comparación de resultados

\subsection{Validación de los modelos}

Luego de la realización de la estimación de los parámetros, lo último es evaluar la validez del modelo. Por lo tanto, la validación se puede realizar por:

5.4.1. Simulación. Mediante MATLAB vamos a realizar una comparación de las salidas de la planta y la de los modelos encontrados, la misma que nos van a mostrar cuál de las funciones de transferencia obtenidas por los métodos ya descritos se aproxima más a la real.

Para realizar la validación de los métodos nos valemos de Simulink, en el cual se ingresan las funciones de transferencias encontradas por los métodos descritos. La Figura 25 nos presenta los resultados obtenidos.

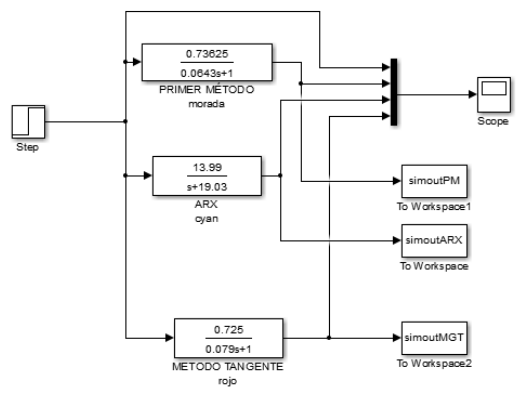

(a). Esquema de simulación

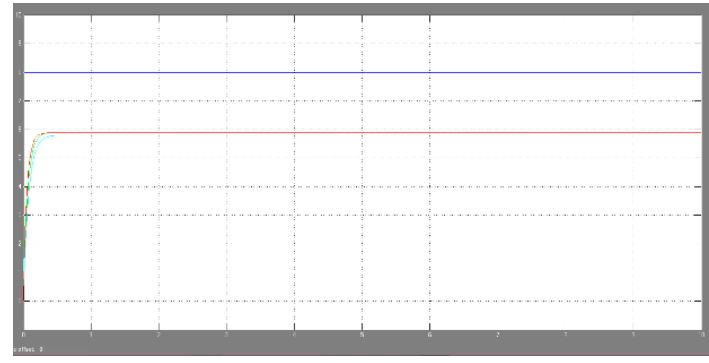

(b). Comparación de resultados

Figura 25. Simulación en MATLAB

Luego para determinar cuál de los métodos se aproxima más a la señal de salida real usamos el archivo de MATLAB CARGAR_DATOS_COM.m el mismo que colocamos a continuación:

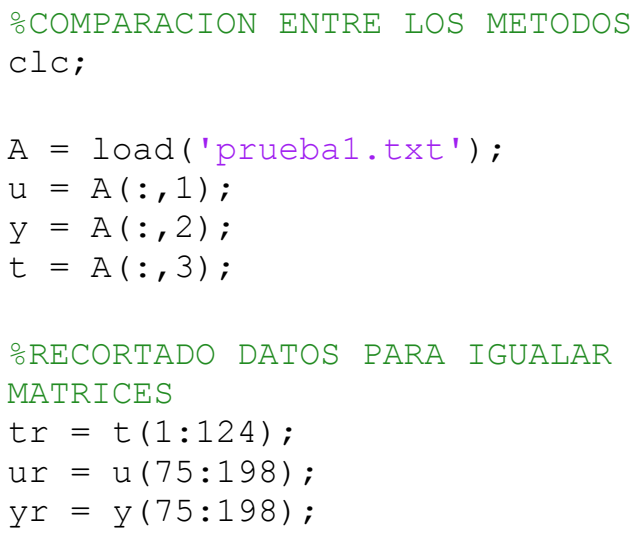

\section{○GRAFICA DE LA RESPUESTA}

subplot $(2,1,1)$;

plot(tr,ur, 'm', tr, yr, 'r', tr, simoutAR

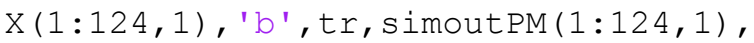
'c',tr, simoutmGT $(1: 124,1)$, 'g')

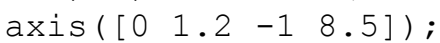

grid on;

legend ('Uk', 'Yk', 'ARX', 'MG', 'MGT') title('VERIFICACIÓN DE LOS MÉTODOS (MÍNIMOS CUADRADOS, MÉTODO GRÁFICO Y MÉTODO DE LA TANGENTE) ') xlabel ('Time') ; ylabel ('Volts')

○GRÁFICA DEL ERROR

eARX $=y r-\operatorname{simoutARX}(1: 124,1)$;

$\mathrm{ePM}=\mathrm{yr}-\operatorname{simoutPM}(1: 124,1) ;$

eMGT = yr - simoutMGT $(1: 124,1)$;

subpl ot $(2,1,2)$;

plot (tr, eARX, 'r', tr, ePM, 'c', tr, eMGT, ' $\mathrm{b}$ ')

$\operatorname{axis}\left(\left[\begin{array}{llll}0 & 1.2 & -0.6 & 1\end{array}\right]\right)$;

grid on;

legend ('Error ARX',

'ErrorMG' , 'ErrorMGT' )

title('COMPARACIÓN DEL ERROR DE LOS MÉTODOS (MÍNIMOS CUADRADOS, MÉTODO GRÁFICO Y MÉTODO DE LA TANGENTE) ') xlabel ('Time'); ylabel('Error') 
La Figura 26 muestra las gráficas que se obtuvieron con este programa, la gráfica de la parte superior nos muestra una comparación entre la salida real y el modelo matemático de los métodos encontrados, la gráfica inferior nos indica un contraste entre los errores de la señal real con cada uno de los métodos encontrados.

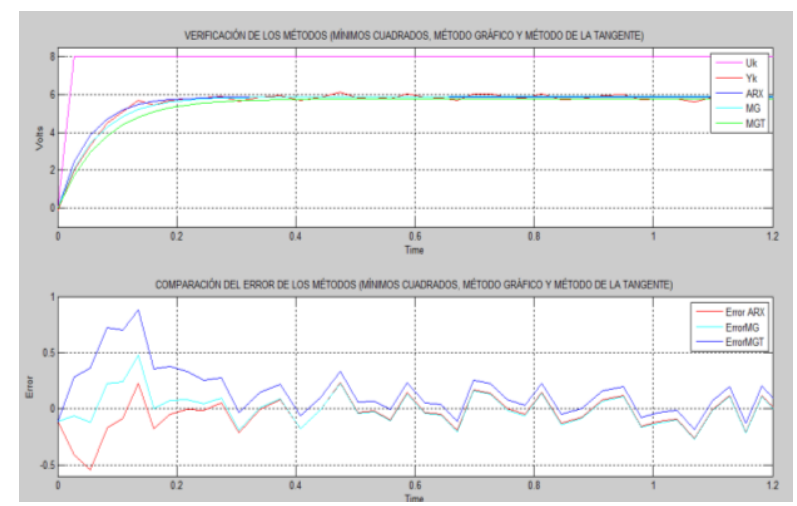

Figura 26. Resultados comparativos de las salidas y los errores

5.4.2. Análisis de Resultados. Se ha procedido a realizar un análisis de resultados de los tres métodos desarrollados dando valores a las diferentes funciones de transferencia, se pudo determinar que el método que menos error posee es el de mínimos cuadrados (ARX) con un valor de $0.27 \%$, seguido del método gráfico de primer orden puro con $1.4112 \%$ y por último el método gráfico de la tangente con un 12.660\%. La Figura 27 muestra las gráficas de las respuestas de cada uno de los modelos a una entrada escalón.

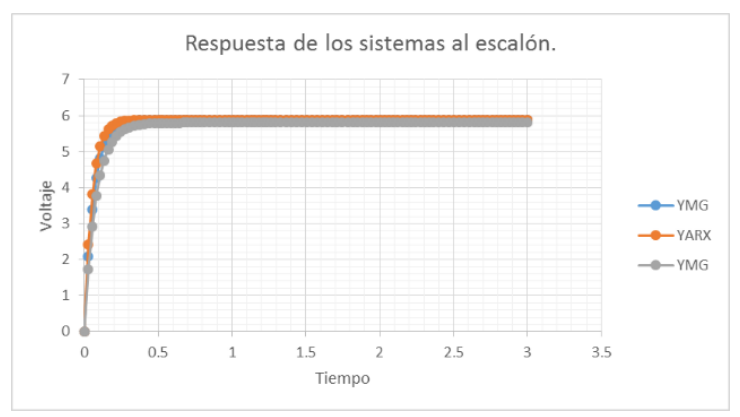

Figura 27. Resultados comparativos de las salidas

\section{Conclusiones}

Las aplicaciones de los métodos de identificación de sistemas son eficientes para modelar la planta, pero se tiene que analizar el proceso para aplicar la técnica adecuada. En este caso los tres métodos empleados son óptimos, confiables y adecuados ya que se aproxima bastante al real, mostrando un buen resultado los algoritmos de identificación; sin embargo, de acuerdo a los resultados analizados por el error podemos decir que el método de Mínimos Cuadrados (ARX110) es el que más se asemeja a la señal de respuesta.

Al ser una planta no muy compleja para su modelación, esta permitió poder realizar más de un método y así poder analizar resultados.

Es claro que con un par de ajustes a los métodos gráficos se hubiese podido obtener mejores resultados, es decir, reducir el error y mejorar la aproximación a la respuesta.

Los métodos gráficos se pueden aplicar de una manera sencilla siempre y cuando el proceso de registro de datos de salida sea confiable.

Los métodos expuestos en este trabajo llevan a concluir que plantas similares a la expuesta en este trabajo no debería ser un problema identificarlas. Si bien es cierto el software y hardware de MatLab y Labview ayudan a simplificar el trabajo, no se puede dejar a un lado la teoría que debe conocer previamente.

\section{Referencias}

[1] Cristian Kunusch, "Identificación de Sistemas Dinámicos", Facultad de Ingeniería -Dpto. Electrotecnia, Universidad Nacional de la Plata, 2003.

[2] Ljung, Lennart, "System identification", John Wiley \& Sons, Inc., 1999.

[3] Santiago Garrido, "Identificación, Estimación y Control de Sistemas No-lineales mediante RGO", Departamento de Ingeniería de Sistemas y Automática, Universidad Carlos III de Madrid, Leganés, 1999.

[4] Dr. Eliezer Colina, "Cátedra de Identificación de Sistemas”, Maestría de Control y Automatización Industrial, Universidad Politécnica Salesiana Sede Cuenca, Febrero 2015.

[5] Ángel Martínez Bueno, "Identificación experimental de sistemas", Sistemas de Control BuenoAutomático, Universidad de Alicante, 2011GITE-IEA.Dr. Pedro Arafet Padilla, Dr. Francisco Chang Mumañ, MSc. Miguel Torres Alberto, MSc. Hugo Dominguez Abreu, "Métodos de Identificación dinámica", Facultad de Ingeniería Eléctrica, Universidad de Oriente, Junio 2008.

[6] Dr. Ismael Minchala, "Cátedra de Identificación de Sistemas-Sesión 1", Maestría de Control y Automatización Industrial, Universidad Politécnica Salesiana Sede Cuenca, Febrero 2015. 